\title{
Allocazione di reni HCV positivi: esperienza internazionale
}

\author{
E. Bertoni, G. Rosso, M. Salvadori \\ U.O. Nefrologia dei Trapianti \\ Azienda Ospedaliero-Universitaria Careggi - Firenze
}

D

ata la carenza degli organi per trapianto, è sempre più diffuso il ricorrere a donatori marginali. Fra tali donatori ci sono anche i pazienti portatori di virus C per l'epatite.

Se utilizzare o meno tali organi è da tempo oggetto di discussioni internazionali. Il non uso porta per molti Paesi a un non utilizzo di circa il $4 \%$ degli organi.

Scopo di questa revisione è quello di mostrare cosa avviene in un Paese come gli Stati Uniti, dotato di validi registri, come USRDS e UNOS. In tal modo rappresentano forse la fonte più ricca di esperienza in un campo particolare come questo.

Le dimensioni del problema sono evidenziate nella Tabella I.

Nel periodo 1996-2001 sono stati utilizzati negli USA complessivamente 873 reni da donatori HCV+: 593 reni sono andati a riceventi $\mathrm{HCV}+, 280$ reni sono stati utilizzati da riceventi HCV- (1). Nel trattare l'argomento seguiremo i criteri esposti nella Tabella II.

\section{Prevalenza dell'infezione $\mathrm{HCV}$ fra i donatori cadavere}

Prendendo in esame i dati delle diverse banche di organi americane, basan- dosi sulla presenza di anticorpi anti HCV nel sangue, i possibili donatori $\mathrm{HCV}+$ sono mediamente il $4 \%$. Probabilmente testando tutti i donatori possibili per RNA virale la percentuale calerebbe ancora, ma anche pazienti

RNA- sono infettanti e comunque i test per RNA virale sono non fattibili nelle 6 ore dell'osservazione. Ci si deve quindi basare su test di dosaggio degli anticorpi, naturalmente di ultima generazione.

TABELLA I - HCV E TRAPIANTO RENALE - DIMENSIONI DEL PROBLEMA

\begin{tabular}{lccc}
\hline \multicolumn{1}{l}{ Totale } & Donatori HCV- & Donatori HCV+ \\
\hline Totale & $36,956(100 \%)$ & $36,083(97.6 \%)$ & $873(2.4 \%)$ \\
& $34,431(93.2 \%)$ & $34,151(94.6 \%)$ & $280(32.1 \%)$ \\
Riceventi HCV - & $2,525(6.8 \%)$ & $1,932(5.4 \%)$ & $593(67.9 \%)$ \\
Riceventi HCV + & & &
\end{tabular}

TABELLA II - RICEVENTI DI ORGANI DA DONATORI HCV+ HANNO RISCHIO DI MORTE PIÙ ALTO

\begin{tabular}{lccc}
\hline & N. & Mortalità & RR Univariato \\
\hline $\mathrm{D}+/ \mathrm{R}-$ & $280(0.8 \%)$ & $25.4 \%$ & $2.49(1.90-3.26)$ \\
$\mathrm{D}+/ \mathrm{R}+$ & $593(1.6 \%)$ & $16.9 \%$ & $1.49(1.20-1.85)$ \\
$\mathrm{D}-/ \mathrm{R}+$ & $1,932(5.2 \%)$ & $15.5 \%$ & $1.38(1.24-1.55)$ \\
$\mathrm{D}-/ \mathrm{R}-$ & $34,431(92.4 \%)$ & $11.8 \%$ & 1.00 \\
Totale & $36,956(100 \%)$ & $12.3 \%$ & \\
\hline
\end{tabular}



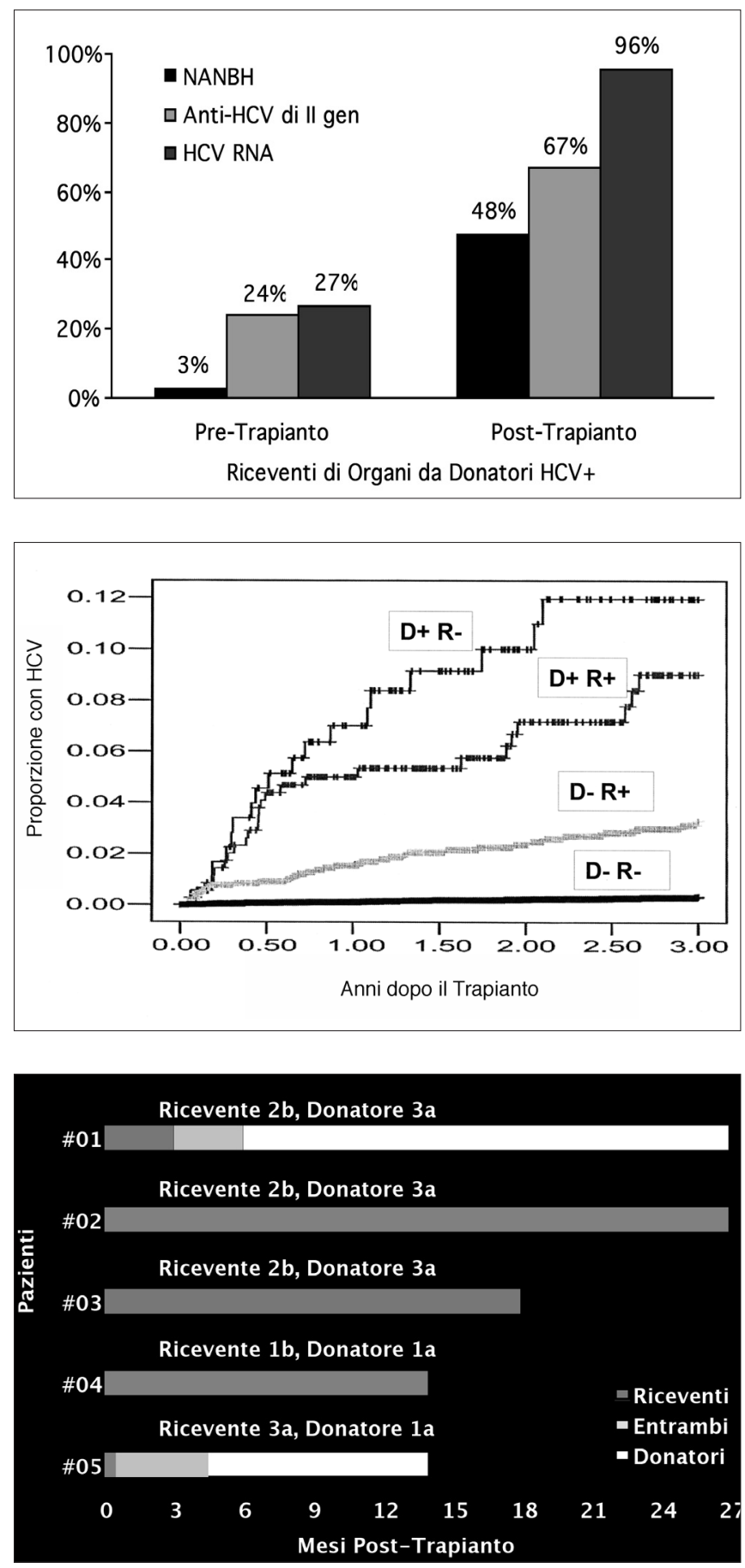

TABELLA III - PAZIENTI TRAPIANTATI CON RENI DA DONATORI HCV+ VANNO MEGLIO DI QUELLI CHE RESTANO IN LISTA D’ATTESA

\begin{tabular}{lcc}
\hline Trapianti renali & $\mathrm{p}$ & $\mathrm{RR}(95 \% \mathrm{CI})$ \\
Trapianti da donatori HCV + vs. Dialisi & 0.25 & $0.76(0.60-0.96)$ \\
Trapianti da donatori cadavere vs. Dialisi & $<0.001$ & $0.47(0.44-0.50)$
\end{tabular}

Fig. 1 - La trasmissione di $H C V$ con trapianto di organi da donatori $\mathrm{HCV}+$ è certa.

Fig. 2 - Riceventi di organi da donatori $\mathrm{HCV}+$ tendono a sviluppare più facilmente epatopatie post-trapianto.

Fig. 3 - Trapianti di organi venti $\mathrm{HCV}+$ da donatori $\mathrm{HCV}+$ in rice-

\section{Trasmissione dell'infezione}

Trapiantando organi $\mathrm{HCV}+$ in pazienti HCV- la trasmissione del virus è certa.

Infatti considerando la situazione biologica pre e post-trapianto da donatore $\mathrm{HCV}+$ si ha (Fig. 1) forte incremento di epatopatia non A non B, di anticorpi anti HCV e di RNA circolante (2).

Per di più i riceventi di organi da donatori $\mathrm{HCV}+$ sviluppano più facilmente malattia epatica post-trapianto (Fig. 2) (3). Osservando la mortalità post-trapianto, questa è più elevata nei riceventi negativi che ricevono organi da donatori positivi. Invece il ricevente positivo, pur avendo ovviamente una mortalità più elevata rispetto al negativo, non ha un maggior rischio di morte se riceve organi da donatori positivi (Fig. 3) (1).

\section{Impatto dello scarto di organi da donatori $\mathrm{HCV}+$}

Osservando i numeri americani concernenti l'attività di donazione e trapianto si osserva che:

Ogni anno si hanno circa 5000 donatori cadavere, di cui circa 200 (4.2\%) sono prevedibilmente $\mathrm{HCV}+$.

Un non uso totale di tali organi comporterebbe la perdita di 420 reni. Se si considera che negli Stati Uniti sono in lista di attesa più di 60000 pazienti, un non uso totale di tali organi non sembra appropriato.

Il problema quindi si sposta su quali pazienti debbano preferenzialmente ricevere tali organi.

Premesso che è ben documentato che non ha rilevanza il genotipo sia del donatore che del ricevente (4), è in primo luogo da osservare che il trapianto di organi da donatori $\mathrm{HCV}+$ in riceventi $\mathrm{HCV}+$ non aumenta né il rischio di malattia epatica, né il rischio di morte (Fig. 4) (5).

Inoltre è ben documentato che il rischio di morte dei pazienti $\mathrm{HCV}+$ che ricevono organi $\mathrm{HCV}+$ è ridotto del $25 \%$ rispetto a chi rimane in dialisi (Tab. III) (6). 

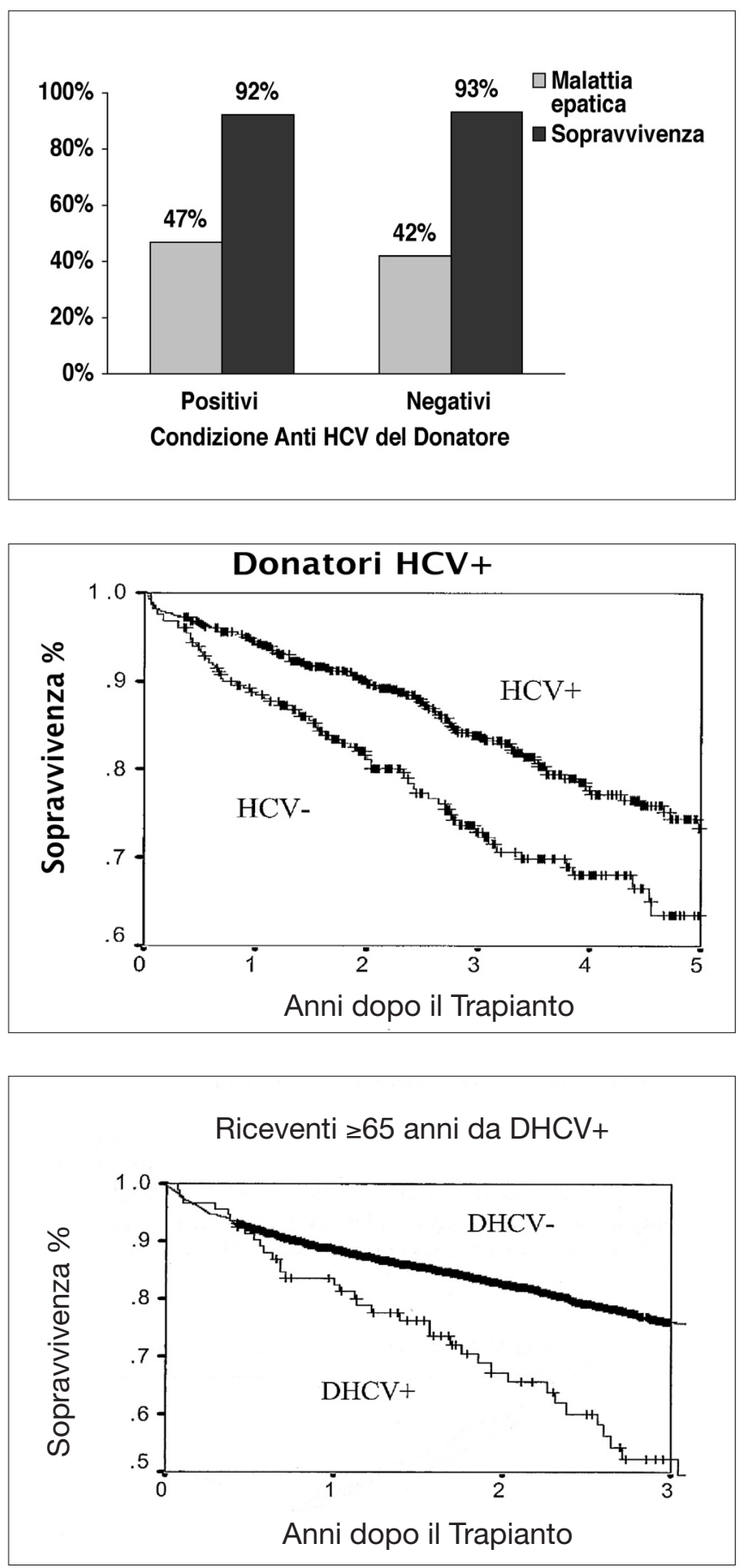

Fig. 4 - Il trapianto di organi da donatori $\mathrm{HCV}+$ in riceventi $\mathrm{HCV}+$ non aumenta il rischio di malattia epatica o morte.

Fig. 5 - Donatori $\mathrm{HCV}+: i$ riceventi $H C V$ - vanno peggio dei riceventi $\mathrm{HCV}+$

Fig. 6 - Riceventi $\geq 65$ anni: riceventi da donatori $\mathrm{HCV}+$ vanno peggio.

\section{Pratica clinica e osservazioni}

Nella pratica clinica americana c'è una tendenza ad allocare reni $\mathrm{HCV}+$, oltre che a pazienti $\mathrm{HCV}+$, anche a riceventi come gli anziani ( $\geq 65$ anni) o di razza afro-americana anche se HCV-. Tale pratica non è assolutamente da raccomandare perché i riceventi $\mathrm{HCV}$ - che ricevono un organo $\mathrm{HCV}+$ hanno un outcome clinico decisamente peggiore rispetto ai pazienti $\mathrm{HCV}+$ (Fig. 5) (1). Il fenomeno è addirittura amplificato in caso di ricevente $\geq 65$ anni (Fig. 6) (1).

\section{Raccomandazioni}

In sintesi gli americani sono giunti a conclusioni assai simili a quelle del Centro Nazionale Trapianti.

In caso di donatori $\mathrm{HCV}+$ è inaccettabile una allocazione degli organi in riceventi non selezionati.

Non è raccomandabile l'allocazione di tali organi in pazienti anziani con poca attesa di vita, perché se ne aumenta il rischio di morte.

Non esistono dati clinici di follow-up esaustivi su cosa accada in riceventi $\mathrm{HCV}+$, ma questa sembra essere l'opzione migliore.

È raccomandabile la realizzazione di registri per monitorizzare l'andamento di tali donazioni, che comunque dovrebbero sempre essere svolte nell'ambito di una corretta informazione di medico e paziente. 


\section{BIBLIOGRAFIA}

1. Abbott KC, Bucci JR, Matsumoto CS, et al. Hepatitis C and Kidney Transplantation in the era of modern immunosuppression. J Am Soc Nephrol 2003; 14: 2908-18.

2. Pereira BJ, Milford EL, Kirkman RL, et al. Prevalence of hepatitis $\mathrm{C}$ virus RNA in organ donors positive for hepatitis $\mathrm{C}$ antibody and in the recipients of their organs. New Engl J Med 1992; 327: 910-5.

3. Abbott KC, Lentine KL, Bucci $\mathrm{JR}$, et al. Impact of diabetes and hepatitis after kidney transplantation on patients who are affected by hepatitis C virus. J Am Soc Nephrol 2004; 15: 3166-74.

4. Widell A, Mansson S, Persson $\mathrm{NH}$, et al. Hepatitis $\mathrm{C}$ superinfection in hepatitis $\mathrm{C}$ virus (HCV)-infected patients transplanted with an HCV-infected kidneys. Transplantation 1995; 60:642-7.

5. Morales JM. 5-year follow-up of recipients with pre-transplant anti HCV. Jam Soc Nephrol 1996; 9: 1936.

6. Abbott KC, Lentine KL, Bucci JR, et al. The impact of transplantation with deceased donors hepatitis C-positive kidneys on survival in wait-listed long-term dialysis patients. Am J Transplant 2004; 4: 2032-7. 